\title{
Systematic analysis of uncertainty principles of the local polynomial Fourier transform
}

\author{
Xiumei $\mathrm{Li}^{1 *}$ and Guoan $\mathrm{Bi}^{2}$
}

\begin{abstract}
In this paper, we show that there are a number of uncertainty principles for the local polynomial Fourier transform and local polynomial periodogram. Systematic analysis of uncertainty principles is given, explicit expressions of the uncertainty relations are derived, and an example using the chirp signal and the Gaussian window function is given to verify the expressions.
\end{abstract}

\section{Introduction}

Time-frequency representations are of significant importance to better describe time-varying signals, i.e, signals with time-varying frequencies. Among the representations, the short-time Fourier transform (STFT) is the simplest and easiest one to implement. However, because the STFT assumes that the frequencies within a signal segment are not changing with time, the resolution in the time and/or frequency domain is often limited for practical applications. To overcome this drawback, the local polynomial Fourier transform (LPFT), as a generalized form of the STFT, was proposed [1]. The kernel of the LPFT uses extra parameters to approximate the signal's phase into a polynomial form. Therefore, the LPFT can describe the time-varying signals with better accuracy, and the resolution representing the signal components in the time-frequency domain can be significantly improved compared to that achieved by the STFT.

The uncertainty principle plays an important role in signal processing [2]. In general, the more concentrated the signal is, the wider band its Fourier transform occupies. It is impossible to arbitrarily concentrate both a timedomain signal and its Fourier transform. This trade-off can be formalized in the form of the uncertainty principle. Similarly, the time-frequency concentration of the transforms belonging to the Cohen class is restricted and related to an uncertainty principle [3]. The STFT is also

\footnotetext{
*Correspondence: lixiumei@pmail.ntu.edu.sg

1 School of Information Science and Engineering, 58 Haishu Road, Hangzhou Normal University, 311121 Hangzhou, China

Full list of author information is available at the end of the article
}

limited by the uncertainty principle [4], and it is understood that a shorter window used to capture the signal segment leads to a poor resolution to represent the signal in the frequency domain, and vice verse. It is not possible to arbitrarily increase the resolution in both domains simultaneously. The standard formulation of the uncertainty principle, known as the global uncertainty principle, is in terms of global standard deviations to involve the entire time range and the entire frequency range of the signal. With regard to the local behavior of the signal, the local uncertainty principle is invoked to present the uncertainty limits on local signal, by defining local quantities as conditional standard deviations [5]. The conditional standard deviations can be considered as the local widths or measures of the local spread in the time and/or frequency domain. The local uncertainty product of the spectrogram and a large class of bilinear time-frequency distributions were considered [5]. It shows that the local uncertainty product of the spectrogram has a lower bound due to the windowing approach and cannot be arbitrarily small, while for a large class of bilinear time-frequency distributions, the local uncertainty product is always less than or equal to the global uncertainty product and can be arbitrarily small.

It has been observed that the resolution of the LPFT in the time-frequency domain is influenced by the window length which controls the trade-off of bias and variance $[1,6]$. A comprehensive study on the uncertainty principle for the LPFT has been reported in [7]. It was shown that when the Gaussian window is used to segment the signal, the uncertainty product of the LPFT is timeindependent if the polynomial coefficients are estimated correctly. However, the work reported in [7] was mainly

\section{Springer}

C $2014 \mathrm{Li}$ and Bi; licensee Springer. This is an Open Access article distributed under the terms of the Creative Commons

Attribution License (http://creativecommons.org/licenses/by/4.0), which permits unrestricted use, distribution, and reproduction in any medium, provided the original work is properly credited. 
focused on the uncertainty product obtained by multiplying the duration and bandwidth of the local signal. Other kinds of uncertainty products of the LPFT, such as the global uncertainty products, have not been reported in the literature.

In this paper, systematic analysis regarding the uncertainty principles of the LPFT is given. Several kinds of uncertainty principles are discussed, including the global uncertainty principle, the uncertainty principle of local duration and conditional standard deviation, the uncertainty principle of local bandwidth and conditional standard deviation, and the uncertainty principle of the conditional standard deviations in time and frequency.

The rest of the paper is organized as follows. After the review on the uncertainty principles of the STFT in Section 2, the characteristic functions of the second-order local polynomial periodogram and the uncertainty principles of the second-order LPFT are discussed in Section 3. Section 4 presents an example of the uncertainty principles of the second-order LPFT by using the chirp signals. Section 5 discusses the uncertainty principles of the Mthorder LPFT. Finally, conclusions are given in Section 6.

\section{Review on the uncertainty principles of STFT}

The uncertainty principles of the STFT were derived in [8], and the definitions and equations will be reviewed in this section.

Let $h(t)$ represent a window function that segments an input signal $s(t)$. By multiplying the input signals with the window function that is peaked around time $t$, the local signal is defined as

$$
s_{t}(\tau)=s(\tau) h(\tau-t) .
$$

The normalized local signal at the time instant $t$ is

$$
\eta_{t}(\tau)=\frac{s(\tau) h(\tau-t)}{\sqrt{P(t)}},
$$

where $P(t)=\int|s(\tau) h(\tau-t)|^{2} d \tau$. For simplicity in the rest of the paper, the integral without limits implies that the integration is from $-\infty$ to $\infty$.

The STFT is the Fourier transform of the local signal

$$
\begin{aligned}
S_{t}(\omega) & =\frac{1}{\sqrt{2 \pi}} \int s_{t}(\tau) e^{-j \omega(\tau-t)} d \tau \\
& =\frac{1}{\sqrt{2 \pi}} \int s(\tau) h(\tau-t) e^{-j \omega(\tau-t)} d \tau .
\end{aligned}
$$

Similarly, the local spectrum is defined as

$$
F_{\omega}(w)=S(w) H(\omega-w),
$$

and the normalized local spectrum is

$$
\mu_{\omega}(w)=\frac{S(w) H(\omega-w)}{\sqrt{P(\omega)}},
$$

where $P(\omega)=\int|S(w) H(\omega-w)|^{2} d w$.
The short-frequency time transform is

$$
s_{\omega}(t)=\frac{1}{\sqrt{2 \pi}} \int S(w) H(\omega-w) e^{j w t} d w .
$$

The Fourier transform pairs of the signal and window are normalized as follows:

$$
\begin{aligned}
S(\omega) & =\frac{1}{\sqrt{2 \pi}} \int s(t) e^{-j \omega t} d t, \\
H(\omega) & =\frac{1}{\sqrt{2 \pi}} \int h(t) e^{-j \omega t} d t .
\end{aligned}
$$

The spectrum of the two-dimensional time-frequency distribution, the spectrogram $P_{S P}(t, \omega)$, is defined as

$$
P_{S P}(t, \omega)=\left|S_{t}(\omega)\right|^{2}=\left|s_{\omega}(t)\right|^{2},
$$

with its marginals expressed as

$$
\begin{aligned}
& P_{S P}(t)=\int P_{S P}(t, \omega) d \omega, \\
& P_{S P}(\omega)=\int P_{S P}(t, \omega) d t .
\end{aligned}
$$

\subsection{Properties of the local signal and spectrum}

The mean time and duration of the normalized local signal in (2) are

$$
\begin{aligned}
\langle\tau\rangle_{t} & =\int \tau\left|\eta_{t}(\tau)\right|^{2} d \tau \\
T_{t}^{2} & =\int\left(\tau-\langle\tau\rangle_{t}\right)^{2}\left|\eta_{t}(\tau)\right|^{2} d \tau .
\end{aligned}
$$

Similarly, from the windowed spectrum, the local frequency and local bandwidth can be defined as follows:

$$
\begin{aligned}
\langle w\rangle_{\omega} & =\int w\left|\mu_{\omega}(w)\right|^{2} d w \\
B_{\omega}^{2} & =\int\left(w-\langle w\rangle_{\omega}\right)^{2}\left|\mu_{\omega}(w)\right|^{2} d w .
\end{aligned}
$$

These quantities pertain to the local signal and local spectrum. They should not be confused with the local properties of the spectrogram which is to be considered in the next subsection. 


\subsection{Local and global quantities for the spectrogram 2.2.1 Local quantities}

The mean averages and conditional standard deviations for the spectrogram are [8]

$$
\begin{aligned}
\langle\omega\rangle_{t} & =\frac{1}{P(t)} \int \omega P_{S P}(t, \omega) d \omega \\
& =\int \eta_{t}^{*}(\tau) \frac{1}{j} \frac{d}{d \tau} \eta_{t}(\tau) d \tau \\
\langle t\rangle_{\omega} & =\frac{1}{P(\omega)} \int t P_{S P}(t, \omega) d t \\
& =\int \mu_{\omega}^{*}(w) \frac{1}{j} \frac{d}{d w} \mu_{\omega}(w) d w \\
\sigma_{\omega \mid t}^{2} & =\frac{1}{P(t)} \int(\omega-\langle\omega\rangle)^{2} P_{S P}(t, \omega) d \omega \\
& =\int \eta_{t}^{*}(\tau)\left(\frac{1}{j} \frac{d}{d \tau}-\langle\omega\rangle_{t}\right)^{2} \eta_{t}(\tau) d \tau \\
& =\int\left|\left(\frac{1}{j} \frac{d}{d \tau}-\langle\omega\rangle_{t}\right) \eta_{t}(\tau)\right|^{2} d \tau \\
\sigma_{t \mid \omega}^{2} & =\frac{1}{P(\omega)} \int(t-\langle t\rangle)^{2} P_{S P}(t, \omega) d t \\
& =\int \mu_{\omega}^{*}(w)\left(\frac{1}{j} \frac{d}{d w}-\langle t\rangle_{\omega}\right)^{2} \mu_{\omega}(w) d w \\
& =\int\left|\left(\frac{1}{j} \frac{d}{d w}-\langle t\rangle_{\omega}\right) \mu_{\omega}(w)\right|^{2} d w .
\end{aligned}
$$

\subsubsection{Global quantities}

The spectrogram is constructed from the signal under observation and the window function. Their mean times and bandwidth of the signal and the window function are [8]

$$
\begin{aligned}
\langle t\rangle^{s} & =\int t|s(t)|^{2} d t \\
\langle t\rangle^{h} & =\int t|h(t)|^{2} d t \\
\langle\omega\rangle^{s} & =\int \omega|S(\omega)|^{2} d \omega \\
\langle\omega\rangle^{h} & =\int \omega|H(\omega)|^{2} d \omega \\
T_{s}^{2} & =\int\left(t-\langle t\rangle^{s}\right)^{2}|s(t)|^{2} d t \\
T_{h}^{2} & =\int\left(t-\langle t\rangle^{h}\right)^{2}|h(t)|^{2} d t \\
B_{s}^{2} & =\int\left(\omega-\langle\omega\rangle^{s}\right)^{2}|S(\omega)|^{2} d \omega \\
B_{h}^{2} & =\int\left(\omega-\langle\omega\rangle^{h}\right)^{2}|H(\omega)|^{2} d \omega .
\end{aligned}
$$

As a two-dimensional density, the spectrogram also has mean durations and mean standard deviations which are defined as

$$
\begin{aligned}
\langle t\rangle^{S P} & =\int t P_{S P}(t, \omega) d t d \omega \\
\langle\omega\rangle^{S P} & =\int \omega P_{S P}(t, \omega) d t d \omega \\
T_{S P}^{2} & =\int\left(t-\langle t\rangle^{S P}\right)^{2} P_{S P}(t, \omega) d t d \omega \\
B_{S P}^{2} & =\int\left(\omega-\langle\omega\rangle^{S P}\right)^{2} P_{S P}(t, \omega) d t d \omega .
\end{aligned}
$$

It has been shown that these quantities can be expressed using the corresponding quantities of the signal and window as [4]

$$
\begin{aligned}
\langle t\rangle^{S P} & =\langle t\rangle^{(s)}-\langle t\rangle^{h} \\
\langle\omega\rangle^{S P} & =\langle\omega\rangle^{(s)}+\langle\omega\rangle^{h} \\
T_{S P}^{2} & =T_{s}^{2}+T_{h}^{2} \\
B_{S P}^{2} & =B_{s}^{2}+B_{h}^{2} .
\end{aligned}
$$

\section{Uncertainty principles of the second-order LPFT}

The $M$ th-order LPFT of the signal $s(t)$ is defined as [1]:

$$
\begin{aligned}
\mathrm{LPFT}_{s}(t, \omega) \\
=\frac{1}{\sqrt{2 \pi}} \int s(t+\tau) h(\tau) \\
\quad \cdot \exp \left\{-j \omega \tau-j \sum_{m=2}^{M} \omega_{m-1} \tau^{m} / m !\right\} d \tau \\
=\frac{1}{\sqrt{2 \pi}} \int s(\tau) h(\tau-t) \\
\quad \cdot \exp \left\{-j \omega(\tau-t)-j \sum_{m=2}^{M} \omega_{m-1}(\tau-t)^{m} / m !\right\} d \tau,
\end{aligned}
$$

where $h(t)$ is the window function to segment the signal, $M$ is the order of the polynomial function, $\omega_{1}, \omega_{2}, \ldots$, and $\omega_{M-1}$ are the first-order derivative and other higher-order derivative of the instantaneous frequency of the analyzed signal. These parameters can be estimated by using the polynomial time frequency transform [9] or the Lv's distribution [10]. The energy distribution of the LPFT is called the local polynomial periodogram (LPP) which is defined as $\left|\operatorname{LPFT}_{s}(t, \omega)\right|^{2}$. When $M=2$, we can get the secondorder LPFT, which has achieved improved performance in many applications such as radar imaging [11,12], nonstationary interference excision in DSSS communications $[13,14]$, chirp signal detection [15], and source localization and tracking in nonstationary environment [16]. A review on the developments and applications of the LPFT can be referred to $[17,18]$. In the following, we will focus on the uncertainty principles of the second-order LPFT and then generalize to those of the $M$ th-order LPFT.

The characteristic function is a powerful tool for the study and construction of densities [4]. The twodimensional characteristic function of the spectrogram $M_{S P}(\theta, \tau)$ is defined as 


$$
\begin{aligned}
M_{S P}(\theta, \tau) & =\iint e^{j \theta t+j \tau \omega}\left|S_{t}(\omega)\right|^{2} d t d \omega \\
& =A_{s}(\theta, \tau) A_{h}(-\theta, \tau)
\end{aligned}
$$

where

$$
A_{s}(\theta, \tau)=\int s\left(t+\frac{\tau}{2}\right) s^{*}\left(t-\frac{\tau}{2}\right) e^{j \theta t} d t
$$

is the ambiguity function of the signal, and $A_{h}$ is the ambiguity function of the window function.

The distribution function may be obtained from $M_{S P}(\theta, \tau)$ by the Fourier inversion,

$P_{S P}(t, \omega)=\left|S_{t}(\omega)\right|^{2}=\iint M_{S P}(\theta, \tau) e^{-j(\theta t+\tau \omega)} d \theta d \tau$.

Proposition 1. The distribution function of the LPP $P_{\text {LPP }}(t, \omega)$ can be presented as

$$
\begin{aligned}
P_{\mathrm{LPP}}(t, \omega) & =\left|\operatorname{LPFT}_{s}(t, \omega)\right|^{2} \\
& =\iint M_{\mathrm{LPP}}(\theta, \tau) e^{-j(\theta t+\tau \omega)} d \theta d \tau,
\end{aligned}
$$

where $M_{\mathrm{LPP}}(\theta, \tau)$ is the characteristic function of the second-order LPP as

$$
\begin{aligned}
M_{\mathrm{LPP}}(\theta, \tau) & =\iint e^{j \theta t+j \tau \omega}\left|\operatorname{LPFT}_{s}(t, \omega)\right|^{2} d t d \omega \\
& =A_{s}(\theta, \tau) A_{h}\left(\omega_{1} \tau+\theta, \tau\right)
\end{aligned}
$$

We will prove Proposition 1 as follows.

Proof. Let us expand the right hand side of (19).

$$
\begin{aligned}
& \iiint \int h\left(x+\frac{\tau}{2}\right) h^{*}\left(x-\frac{\tau}{2}\right) e^{j\left(\omega_{1} \tau+\theta\right) x} \\
& s\left(y+\frac{\tau}{2}\right) s^{*}\left(y-\frac{\tau}{2}\right) e^{j \theta y} e^{-j \theta t-j \tau \omega} d x d y d \tau d \theta \\
& =\iiint \frac{1}{2 \pi} h\left(x+\frac{\tau}{2}\right) h^{*}\left(x-\frac{\tau}{2}\right) e^{j\left(\omega_{1} \tau\right) x} \\
& s\left(y+\frac{\tau}{2}\right) s^{*}\left(y-\frac{\tau}{2}\right) e^{-j \tau \omega} \delta(t-x-y) d x d y d \tau \\
& =\iint h\left(x+\frac{\tau}{2}\right) h^{*}\left(x-\frac{\tau}{2}\right) e^{j\left(\omega_{1} \tau\right) x} \\
& s\left(t-x+\frac{\tau}{2}\right) s^{*}\left(t-x-\frac{\tau}{2}\right) e^{-j \tau \omega} d x d \tau .
\end{aligned}
$$

Let $a=x+\frac{\tau}{2}$ and $b=x-\frac{\tau}{2}$, then

$$
x=\frac{a+b}{2}, \quad \tau=a-b .
$$

With $d \tau d x=|J| d a d b$, where the Jacobian determinant is

$$
J=\left|\begin{array}{ll}
\frac{\partial x}{\partial a} & \frac{\partial x}{\partial b} \\
\frac{\partial \tau}{\partial a} & \frac{\partial \tau}{\partial b}
\end{array}\right|=-1
$$

(21) becomes

$$
\begin{aligned}
& \frac{1}{\sqrt{2 \pi}} \int h(a) s^{*}(t-a) \exp (-j a \omega) \exp \left(j a^{2} \frac{\omega_{1}}{2}\right) d a \\
& \quad \cdot \frac{1}{\sqrt{2 \pi}} \int h^{*}(b) s(t-b) \exp (j b \omega) \exp \left(-j b^{2} \frac{\omega_{1}}{2}\right) d b \\
& =\left|\mathrm{LPFT}_{s}(t, \omega)\right|^{2} .
\end{aligned}
$$

From Proposition 1, we can easily get the following Corollary 1 including (22) to (24).

Corollary 1. The total energy can also be given by the characteristic function evaluated at zero.

$$
\begin{aligned}
\iint P_{\mathrm{LPP}}(t, \omega) d t d \omega & =M_{\mathrm{LPP}}(0,0) \\
& =A_{s}(0,0) A_{h}(0,0) \\
& =\int|s(t)|^{2} d t \cdot \int|h(t)|^{2} d t .
\end{aligned}
$$

The time marginal is obtained by integrating over frequency,

$$
\begin{aligned}
P_{\mathrm{LPP}}(t) & =\int P_{\mathrm{LPP}}(t, \omega) d \omega \\
& =\int|L(\tau)|^{2}|h(\tau-t)|^{2} d \tau,
\end{aligned}
$$

where $L(\tau)=s(\tau) e^{j \omega t} e^{-j \frac{\omega_{1}}{2}(\tau-t)^{2}}$.

Similarly, the frequency marginal is

$$
\begin{aligned}
P_{\mathrm{LPP}}(\omega) & =\int P_{\mathrm{LPP}}(t, \omega) d t \\
& =\int\left|S^{\prime}(w)\right|^{2}|H(\omega-w)|^{2} d w,
\end{aligned}
$$

where $S^{\prime}(w)$ is the Fourier transform of signal $L(\tau)$.

Since the second-order LPFT can be considered as the STFT with modulated window function, it is expected that uncertainty limits for LPP can be derived from corresponding SP limits, that is

$$
P_{\mathrm{LPP}: S}(t, \omega)=P_{S P: L}(t, \omega) .
$$

Therefore, we can use the uncertainty principles of the STFT for signal $L(\tau)$ to discuss the uncertainty principles of the LPFT for signal $s(\tau)$.

It should be noted that the subscript LPP:s has the same meaning as the subscript ${ }_{\text {LPP }}$, which means the LPP for the signal $s(\tau)$. The subscript ${ }_{S P}$ has the same meaning as the subscript $S P$ :s, which means the SP for the signal $s(\tau)$. The subscript LPP:s is used together with subscript $S P: L$ with the same meaning, to indicate the LPP of signal $s(\tau)$ and the spectrogram of signal $L(\tau)$, respectively.

We apply the results and equations in [6] to the case in the LPFT/LPP and get the uncertainty principles of the LPFT/LPP with the Gaussian window as shown in Table 1. Details of the uncertainty principles are discussed as follows. 
The first type of uncertainty principle: the global uncertainty principle

Considering signal $L(\tau)$ and the window $h(t)$ as two separate functions, each satisfying the uncertainty principle [4]

$$
\begin{aligned}
& T_{L}^{2} B_{L}^{2} \geq \frac{1}{4}, \\
& T_{h}^{2} B_{h}^{2} \geq \frac{1}{4} .
\end{aligned}
$$

Now, we consider the uncertainty principle for the global duration and spread of the spectrogram of signal $L(\tau)$, which is the LPP of the signal $s(\tau)$. We can relate the global uncertainty product of the LPP to the uncertainties of the signal and window. Therefore, we have

$$
\begin{aligned}
& T_{\mathrm{LPP}}^{2} B_{\mathrm{LPP}}^{2} \\
& =\left(T_{L}^{2}+T_{h}^{2}\right)\left(B_{L}^{2}+B_{h}^{2}\right) \\
& =T_{L}^{2} B_{L}^{2}+T_{h}^{2} B_{h}^{2}+T_{L}^{2} B_{h}^{2}+T_{h}^{2} B_{L}^{2} .
\end{aligned}
$$

Using (26) and noting that the last two terms in (27) are manifestly positive, we always have that

$$
T_{\mathrm{LPP}}^{2} B_{\mathrm{LPP}}^{2} \geq \frac{1}{2} \text {. }
$$

However, the last two terms in (27) can never be zero, and hence we could obtain a stronger inequality. Assume that we have a Gaussian signal and window. In this case, $T_{L}^{2} B_{L}^{2}=\frac{1}{4}$ and $T_{h}^{2} B_{h}^{2}=\frac{1}{4}$ and therefore,

$$
T_{\mathrm{LPP}}^{2} B_{\mathrm{LPP}}^{2}=\frac{1}{2}+\frac{1}{4}\left(\frac{T_{L}^{2}}{T_{h}^{2}}+\frac{T_{h}^{2}}{T_{L}^{2}}\right) .
$$

Because the minimum is achieved when $T_{L}^{2}=T_{h}^{2}$, the global uncertainty principle for the LPP of signal $s(t)$ becomes

$$
T_{\mathrm{LPP}}^{2} B_{\mathrm{LPP}}^{2} \geq 1 \text {. }
$$

The second type of uncertainty principle: local duration-conditional standard deviation

For the second-order LPFT, the normalized local signal,

$$
\eta_{t}(\tau)=\frac{s(\tau) h(\tau-t) \exp \left\{j \omega t-j \frac{\omega_{1}}{2}(\tau-t)^{2}\right\}}{\sqrt{P(t)}},
$$

Table 1 Expressions of the uncertainty principles for the second-order LPFT

\begin{tabular}{ll}
\hline Type of uncertainty principle & Uncertainty product \\
\hline 1. Global uncertainty principle & $T_{\mathrm{LPP}}^{2} B_{\mathrm{LPP}}^{2} \geq 1$ \\
$\begin{array}{l}\text { 2. Local duration-conditional standard } \\
\text { deviation }\end{array}$ & $\sigma_{\omega \mid t}^{2} T_{t}^{2} \geq \frac{1}{4}$ \\
$\begin{array}{l}\text { 3. Local bandwidth-conditional standard } \\
\text { deviation }\end{array}$ & $\sigma_{t \mid \omega}^{2} B_{\omega}^{2} \geq \frac{1}{4}$ \\
$\begin{array}{l}\text { 4. Conditional standard deviations in time } \\
\text { and frequency }\end{array}$ & $\sigma_{t \mid \omega}^{2} \sigma_{\omega \mid t}^{2}>\frac{1}{4}$ \\
\hline
\end{tabular}

which has $T_{t}$ as its duration, and $P(t)=\int|s(\tau) h(\tau-t)|^{2} d \tau$.

The Fourier transform of the local signal is

$$
F_{t}(\omega)=\frac{1}{\sqrt{2 \pi}} \int \eta_{t}(\tau) \exp \{-j \omega \tau\} d \tau,
$$

whose bandwidth is

$$
\begin{aligned}
& \int\left(\omega-\langle\omega\rangle_{t}\right)^{2}\left|F_{t}(\omega)\right|^{2} d \omega \\
& =\frac{1}{P(t)} \int\left(\omega-\langle\omega\rangle_{t}\right)^{2}\left|S_{t}(\omega)\right|^{2} d \omega \\
& =\sigma_{\omega \mid t}^{2} .
\end{aligned}
$$

That is, the bandwidth of the local signal is the conditional standard deviation of the LPP. Hence, the second type of uncertainty principle is expressed as

$$
\sigma_{\omega \mid t}^{2} T_{t}^{2} \geq \frac{1}{4}
$$

The third type of uncertainty principle: local bandwidth-conditional standard deviation

The local spectrum is defined as

$$
F_{\omega}(w)=S^{\prime}(w) H(\omega-w) .
$$

The normalized local spectrum is

$$
\mu_{\omega}(w)=\frac{S^{\prime}(w) H(\omega-w)}{\sqrt{P(\omega)}},
$$

where $P(\omega)=\int\left|S^{\prime}(w) H(\omega-w)\right|^{2} d w$. The local spectrum has the following signal as its Fourier transform

$$
f_{\omega}(t)=\frac{1}{\sqrt{2 \pi}} \frac{1}{\sqrt{P(\omega)}} \int S^{\prime}(w) H(\omega-w) e^{j w t} d w .
$$

The bandwidth of this signal is $B_{\omega}$ as given in (11). Its duration is

$$
\begin{aligned}
& \int\left(t-\langle t\rangle_{\omega}\right)^{2}\left|f_{\omega}(t)\right|^{2} d t \\
& =\frac{1}{P(\omega)} \int\left(t-\langle t\rangle_{\omega}\right)^{2}\left|s_{\omega}(t)\right|^{2} d t \\
& =\sigma_{t \mid \omega}^{2},
\end{aligned}
$$

which is the conditional standard deviation of time for a given frequency of the LPP. Therefore, we have

$$
\sigma_{t \mid \omega}^{2} B_{\omega}^{2} \geq \frac{1}{4}
$$

The fourth type of uncertainty principle: conditional standard deviations in time and frequency

We now try to obtain the uncertainty relation which directly relates the two conditional standard deviations of the LPP, $\sigma_{t \mid \omega}^{2}$ and $\sigma_{\omega \mid t}^{2}$. Following the procedure in [8], we get

$\sigma_{t \mid \omega}^{2} \sigma_{\omega \mid t}^{2} \geq \int\left(\frac{d}{d \tau}\left|\eta_{t}(\tau)\right|\right)^{2} d \tau \int\left(\frac{d}{d w}\left|\mu_{\omega}(w)\right|\right)^{2} d w,(38)$ 
which is called the local uncertainty product in [5]. Following the procedure in [5], the local uncertainty product of the LPP for a Gaussian window is achieved as

$$
\sigma_{t \mid \omega}^{2} \sigma_{\omega \mid t}^{2}>\frac{1}{4}
$$

It is shown in [5] that for the general case as well as a large subset of bilinear distributions such as WignerVille distribution, the local uncertainty product is upper bounded by the global uncertainty product, and it can be arbitrarily small even though the product of the global variance cannot. However, for the STFT/spectrogram, the local uncertainty product cannot be arbitrarily small, and there is a lower bound on the local uncertainty product of the spectrogram due to the windowing operation. This limitation is an inherent property of the spectrogram and is not a property of the signal or a fundamental limit. Since the LPFT/LPP also uses the windowing operation, its local uncertainty product has a lower bound as the spectrogram. In Section 4, examples are given to show that the local uncertainty product of the LPFT/LPP has a lower bound and cannot be arbitrarily small.

\section{Example}

Let us consider an example to verify the expressions in Section 3 by using the chirp signal

$$
s(t)=\left(\frac{\alpha}{\pi}\right)^{1 / 4} \exp \left\{\frac{-\alpha t^{2}}{2}+j a_{0} t+\frac{j a_{1} t^{2}}{2}\right\},
$$

as the input signal of the second-order LPFT. A Gaussian window is used to obtain the segments of the signal $s(t)$

$$
h(t)=\left(\frac{a}{\pi}\right)^{1 / 4} \exp \left\{-\frac{a t^{2}}{2}\right\}
$$

where $a>0$ is the parameter to control the window width.

Therefore, we have

$$
\begin{aligned}
L(\tau)= & s(\tau) \exp \{j \omega t\} \exp \left\{-j \frac{\omega_{1}}{2}(\tau-t)^{2}\right\} \\
= & \left(\frac{\alpha}{\pi}\right)^{1 / 4} \exp \left\{\frac{-\alpha \tau^{2}}{2}+j a_{0} \tau+\frac{j a_{1} \tau^{2}}{2}\right\} \\
& \cdot \exp \left\{j \omega t-j \frac{\omega_{1}}{2}(\tau-t)^{2}\right\} .
\end{aligned}
$$

According to the definitions in (23) and (24), we can calculate that

$$
\begin{aligned}
P_{\mathrm{LPP}}(t) & =\int|L(\tau) h(\tau-t)|^{2} d \tau \\
& =\left(\frac{a \alpha}{\pi(a+\alpha)}\right)^{1 / 2} \exp \left[-\frac{a \alpha}{a+\alpha} t^{2}\right], \\
P_{\mathrm{LPP}}(\omega) & =\int\left|S^{\prime}(w) H(\omega-w)\right|^{2} d w \\
& =\left(\frac{a^{\prime} \alpha^{\prime}}{\pi\left(a^{\prime}+\alpha^{\prime}\right)}\right)^{1 / 2} \exp \left[-\frac{a^{\prime} \alpha^{\prime}}{a^{\prime}+\alpha^{\prime}}\left(\omega-a_{0}-\omega_{1} t\right)^{2}\right] .
\end{aligned}
$$

with

$$
\begin{aligned}
\alpha^{\prime} & =\frac{\alpha}{\alpha^{2}+\left(a_{1}-\omega_{1}\right)^{2}} \\
\beta^{\prime} & =\frac{a_{1}-\omega_{1}}{\alpha^{2}+\left(a_{1}-\omega_{1}\right)^{2}} \\
a^{\prime} & =\frac{1}{a}
\end{aligned}
$$

Since

$\left|\eta_{t}(\tau)\right|^{2}=\left(\frac{\alpha+a}{\pi}\right)^{1 / 2} \exp \left\{-(\alpha+a)\left(\tau-\frac{a t}{\alpha+a}\right)^{2}\right\}$,

the spectra of the signal and the window are

$$
\begin{aligned}
S^{\prime}(\omega)= & \exp \left\{j \omega t-j \frac{\omega_{1}}{2} t^{2}\right\} \\
& \sqrt{\frac{\sqrt{\alpha / \pi}}{\alpha-j a_{1}}} \exp \left\{-\frac{\left(\omega-a_{0}-\omega_{1} t\right)}{2\left(\alpha-j a_{1}\right)}\right\}, \\
H(\omega)= & \sqrt[4]{1 / a \pi} \exp \left\{-\frac{\omega^{2}}{2 a}\right\} .
\end{aligned}
$$

Therefore,

$$
\begin{aligned}
& \left|S^{\prime}(\omega)\right|=\left(\alpha^{\prime} / \pi\right)^{1 / 4} \exp \left\{-\frac{1}{2} \alpha^{\prime}\left(\omega-a_{0}-\omega_{1} t\right)^{2}\right\}, \\
& |H(\omega)|=\left(a^{\prime} / \pi\right)^{1 / 4} \exp \left\{-\frac{1}{2} a^{\prime} \omega^{2}\right\} .
\end{aligned}
$$

By using these relations and definitions from (10) to (12), and using $P_{\mathrm{LPP}}(t)$ and $P_{\mathrm{LPP}}(\omega)$ for calculation, we obtain

$$
\begin{aligned}
\langle\tau\rangle_{t} & =\frac{a}{a+\alpha} t \\
T_{t}^{2} & =\frac{1}{2(a+\alpha)} \\
\langle\omega\rangle_{t} & =\frac{a\left(a_{1}-\omega_{1}\right)}{a+\alpha} t+a_{0}+\omega_{1} t \\
\sigma_{\omega \mid t}^{2} & =\frac{1}{2}(a+\alpha)+\frac{1}{2} \frac{\left(a_{1}-\omega_{1}\right)^{2}}{a+\alpha} \\
\langle w\rangle_{\omega} & =\frac{a^{\prime} \omega+\alpha^{\prime}\left(a_{0}+\omega_{1} t\right)}{a^{\prime}+\alpha^{\prime}} \\
B_{\omega}^{2} & =\frac{1}{2\left(a^{\prime}+\alpha^{\prime}\right)} \\
\langle t\rangle_{\omega} & =\frac{a^{\prime} \beta^{\prime}}{a^{\prime}+\alpha^{\prime}}\left(\omega-a_{0}-\omega_{1} t\right) \\
\sigma_{t \mid \omega}^{2} & =\frac{1}{2}\left(a^{\prime}+\alpha^{\prime}\right)+\frac{1}{2} \frac{\beta^{\prime 2}}{a^{\prime}+\alpha^{\prime}} .
\end{aligned}
$$




\section{Global uncertainty principle}

Using (13), we can calculate

$$
\begin{aligned}
T_{L}^{2} & =\frac{1}{2 \alpha} \\
T_{h}^{2} & =\frac{1}{2 a} \\
B_{L}^{2} & =\frac{1}{2 \alpha^{\prime}} \\
B_{h}^{2} & =\frac{1}{2 a^{\prime}} .
\end{aligned}
$$

Therefore, we have

$$
\begin{aligned}
& T_{\mathrm{LPP}}^{2}=\frac{1}{2}\left(\frac{1}{a}+\frac{1}{\alpha}\right) \\
& B_{\mathrm{LPP}}^{2}=\frac{1}{2}\left(\frac{1}{a^{\prime}}+\frac{1}{\alpha^{\prime}}\right)=\frac{1}{2}\left(a+\alpha+\frac{\left(a_{1}-\omega_{1}\right)^{2}}{\alpha}\right) .
\end{aligned}
$$

Then

$T_{\mathrm{LPP}}^{2} B_{\mathrm{LPP}}^{2}=1+\frac{(\alpha-a)^{2}}{4 a \alpha}+\frac{1}{4}\left(\frac{1}{a}+\frac{1}{\alpha}\right) \frac{\left(a_{1}-\omega_{1}\right)^{2}}{\alpha} \geq 1$,

which is consistent with (29). The equality can be achieved when $\alpha=a$, and the parameter $\omega_{1}$ is estimated correctly, that is $\omega_{1}=a_{1}$.

The second type of uncertainty principle

The duration and conditional standard deviations are given by

$$
\begin{aligned}
T_{t}^{2} & =\frac{1}{2(a+\alpha)} \\
\sigma_{\omega \mid t}^{2} & =\frac{1}{2}(a+\alpha)+\frac{1}{2} \frac{\left(a_{1}-\omega_{1}\right)^{2}}{a+\alpha} .
\end{aligned}
$$

Therefore,

$$
\sigma_{\omega \mid t} T_{t}=\frac{1}{2} \sqrt{1+\frac{\left(a_{1}-\omega_{1}\right)^{2}}{(a+\alpha)^{2}}} \geq \frac{1}{2}
$$

which is consistent with the uncertainty principle given in (32).

\section{The third type of uncertainty principle}

We also have the bandwidth and conditional standard deviations as

$$
\begin{aligned}
B_{\omega}^{2} & =\frac{1}{2\left(a^{\prime}+\alpha^{\prime}\right)} \\
\sigma_{t \mid \omega}^{2} & =\frac{1}{2}\left(a^{\prime}+\alpha^{\prime}\right)+\frac{1}{2} \frac{\beta^{2}}{a^{\prime}+\alpha^{\prime}} .
\end{aligned}
$$

Therefore,

$$
\sigma_{t \mid \omega} B_{\omega}=\frac{1}{2} \sqrt{1+\frac{\beta^{\prime 2}}{\left(a^{\prime}+\alpha^{\prime}\right)^{2}}} \geq \frac{1}{2}
$$

which is consistent with the uncertainty principle given in (37).

\section{The fourth type of uncertainty principle}

The fourth type of uncertainty principle deals directly with both conditional standard deviations. It states that

$$
\sigma_{t \mid \omega}^{2} \sigma_{\omega \mid t}^{2} \geq \int\left(\frac{d}{d \tau}\left|\eta_{t}(\tau)\right|\right)^{2} d \tau \int\left(\frac{d}{d w}\left|\mu_{\omega}(w)\right|\right)^{2} d w .
$$

By direct calculation, we have

$$
\begin{aligned}
\int\left(\frac{d}{d \tau}\left|\eta_{t}(\tau)\right|\right)^{2} d \tau & =\frac{1}{2}(a+\alpha) \\
\int\left(\frac{d}{d w}\left|\mu_{\omega}(w)\right|\right)^{2} d w & =\frac{1}{2}\left(a^{\prime}+\alpha^{\prime}\right) .
\end{aligned}
$$

Therefore,

$$
\sigma_{t \mid \omega}^{2} \sigma_{\omega \mid t}^{2} \geq \frac{1}{4}(a+\alpha)\left(a^{\prime}+\alpha^{\prime}\right) .
$$

The exact calculations of $\sigma_{t \mid \omega}^{2}$ for this example are

$$
\begin{aligned}
\sigma_{t \mid \omega}^{2} \sigma_{\omega \mid t}^{2}= & \frac{1}{4}\left[\frac{(a+\alpha)^{2}+\left(a_{1}-\omega_{1}\right)^{2}}{a+\alpha}\right] \\
& \times\left[\frac{\left(a^{\prime}+\alpha^{\prime}\right)^{2}+\beta^{\prime 2}}{a^{\prime}+\alpha^{\prime}}\right],
\end{aligned}
$$

which implies that (50) is satisfied.

As $\alpha$ goes to zero, the signal in (40) becomes a chirp signal with constant amplitude, and the local uncertainty product of the LPP becomes

$$
\sigma_{t \mid \omega}^{2} \sigma_{\omega \mid t}^{2}>\frac{1}{4} \quad \text { as } \quad \alpha \rightarrow 0 .
$$

It can be easily shown that for arbitrary values of the signal parameters, the minimum of the local uncertainty product for the LPP in (51) can be achieved when the Gaussian window width parameter satisfies $a=\sqrt{\alpha^{2}+\left(a_{1}-\omega_{1}\right)^{2}}$.

With different width parameters of the Gaussian window, Figure 1 shows the time-frequency representations achieved by using the second-order LPFT to process the signal consisting of chirp components. Following the definition in (40), the signal components are Gaussianmodulated chirp components. Expressions of the first two chirp components and the second two chirp components are as follows:

$$
\begin{aligned}
s_{1}(t)= & \left(\frac{0.0001}{\pi}\right)^{1 / 4} \exp \left(\frac{-0.0001(t-260)^{2}}{2}\right) \\
& \times \exp \left(j 2 \pi\left(0.5 t-0.000481 t^{2}\right)\right) \\
& +\left(\frac{0.0001}{\pi}\right)^{1 / 4} \exp \left(\frac{-0.0001(t-260)^{2}}{2}\right) \\
& \times \exp \left(j 2 \pi\left(0.4 t-0.000481 t^{2}\right)\right), \\
s_{2}(t)= & \left(\frac{0.0001}{\pi}\right)^{1 / 4} \exp \left(\frac{-0.0001(t-260)^{2}}{2}\right) \\
& \times \exp \left(j 2 \pi\left(0.35 t-0.000481 t^{2}\right)\right) \\
& +\left(\frac{0.0001}{\pi}\right)^{1 / 4} \exp \left(\frac{-0.0001(t-260)^{2}}{2}\right) \\
& \times \exp \left(j 2 \pi\left(0.25 t-0.000481 t^{2}\right)\right),
\end{aligned}
$$




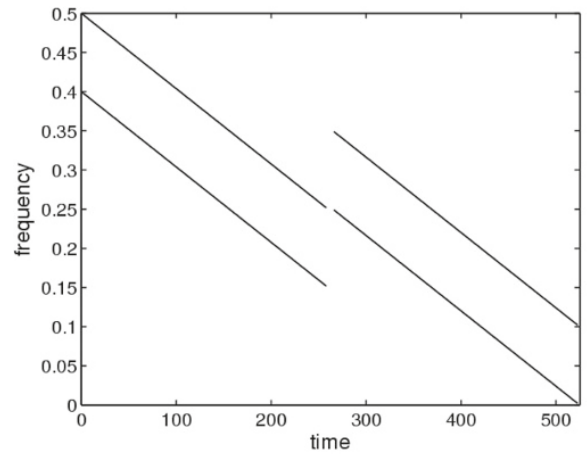

(a) the instantaneous frequency of the chirp signal

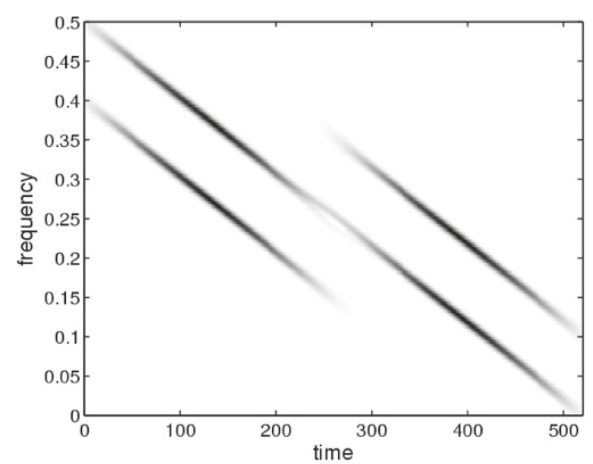

(c) LPP with $a=2.5$

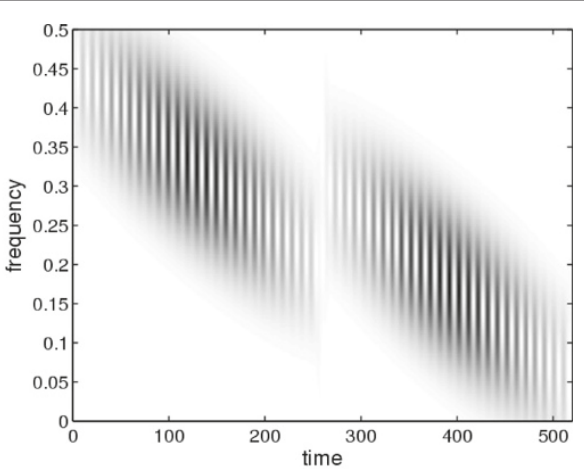

(b) LPP with $a=25$

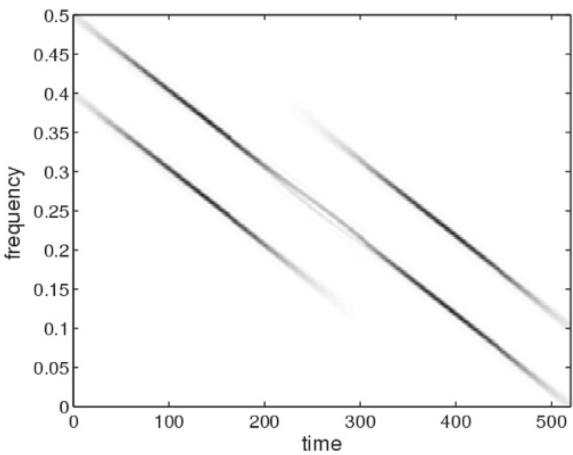

(d) LPP with $a=0.25$

Figure 1 The second-order LPFT of a chirp signal with different window width parameter $\boldsymbol{a}$. The image shows the instantaneous frequency of the chirp signal and the LPP with different window width parameter $a$. (a) The instantaneous frequency of the chirp signal; (b) LPP with $a=25$; (c) LPP with $a=2.5$; (d) LPP with $a=0.25$.

and there are five-point spaces between $s_{1}(t)$ and $s_{2}(t)$. Without loss of generality, the sampling interval is assumed to be unit. In Figure 1, truncated Gaussian window is used, and the window length is $1 / 4$ of the signal length. It is seen that as $a$ decreases or the window width increases, the chirp components become more concentrated in the frequency direction, or equivalently, the resolution of the signal representation in the frequency direction is increased. As for the resolution in the time direction, the signal components in Figure $1 \mathrm{~b}$ can be clearly separated in the time instant 260 . As the parameter $a$ decreases, such separation in time direction disappears. Therefore, from Figure 1b,d, we can observe that the resolution in the time direction decreases as the parameter $a$ decreases. This observation is consistent with the derivation in (45). For example, decreasing the window parameter $a$ leads to the increasing of the signal duration $T_{t}^{2}$, or equivalently, the decreasing of signal time resolution, as shown in Figure 2a,b,c by sampling the LPP at a particular frequency instant $f=0.25$. At the same time, the conditional standard deviation $\sigma_{\omega \mid t}^{2}$ decreases as $a$ decreases so that the signal frequency resolution is increased, as shown in Figure 2d,e,f by sampling the LPP at a particular time instant $t=400$.

\section{Uncertainty principle of the Mth-order LPFTs}

The uncertainty product can be similarly derived for higher-order LPFTs whose input signals are the same order polynomial phase signals, as shown in Table 2. For simplicity, only the major steps of derivation are presented in this section.

Let us consider an $M$ th-order polynomial phase signal defined as

$$
s(t)=\left(\frac{\alpha}{\pi}\right)^{1 / 4} e^{-\alpha t^{2} / 2} \exp \left\{j \sum_{m=1}^{M} \frac{a_{m-1} t^{m}}{m !}\right\},
$$

where the phase of the signal is

$$
\Phi(t)=\sum_{m=1}^{M} \frac{a_{m-1} t^{m}}{m !} .
$$

Because the local signal segment is

$$
L(\tau)=s(\tau) e^{j \omega t} e^{-j \sum_{m=2}^{M} \frac{\omega_{m-1}}{m !}(\tau-t)^{m}},
$$

we have

$$
\left|S^{\prime}(\omega)\right|=\left(\frac{1}{\pi \alpha}\right)^{\frac{1}{4}} e^{-\frac{\left(\omega-j \sum_{m=2}^{M} \frac{\omega_{m-1}}{(m-1) !} !^{m-1}\right)^{2}}{2 \alpha}} .
$$




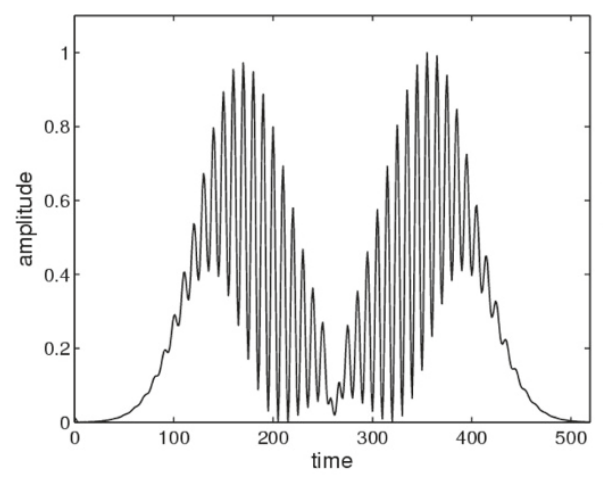

(a) time resolution, $\mathrm{a}=25$

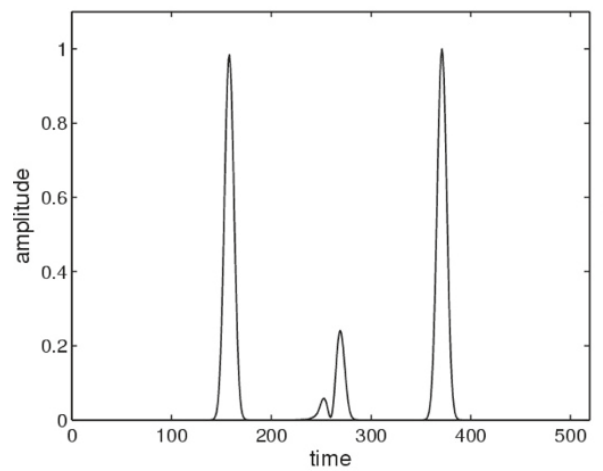

(b) time resolution, $\mathrm{a}=2.5$

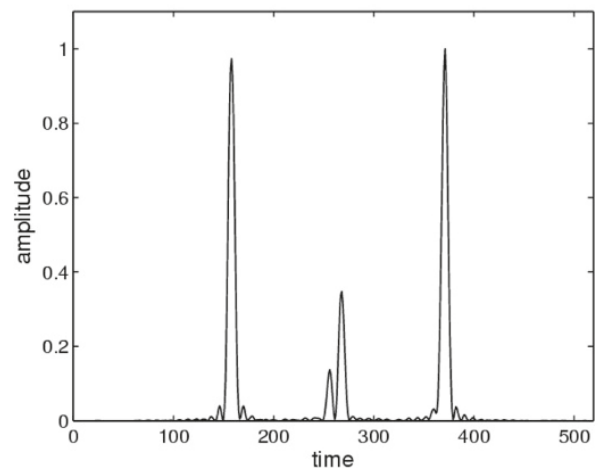

(c) time resolution, $\mathrm{a}=0.25$

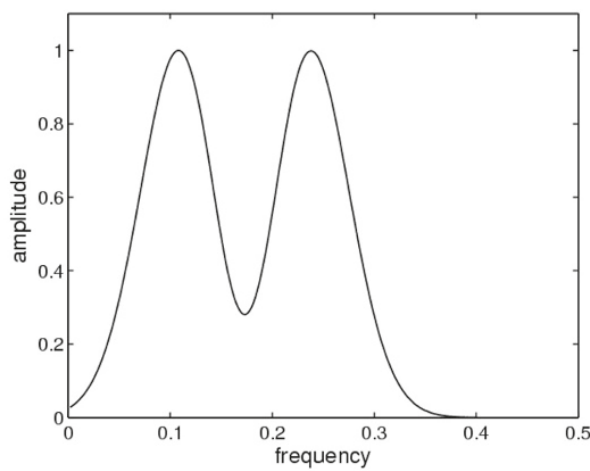

(d) frequency resolution, $a=25$

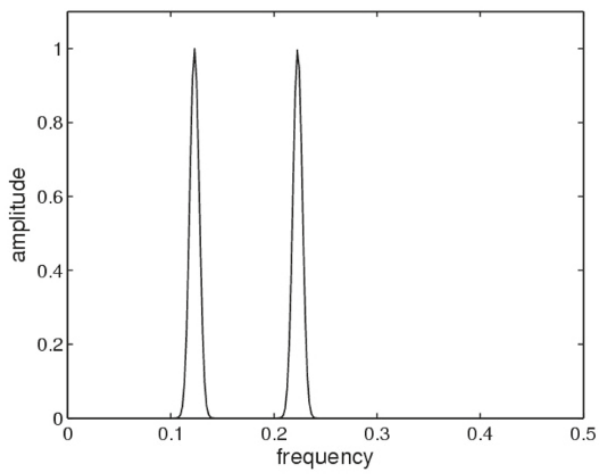

(e) frequency resolution, $a=2.5$

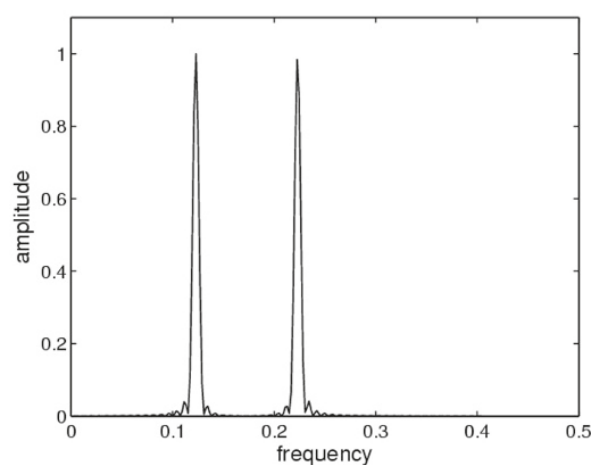

(f) frequency resolution, $a=0.25$

Figure 2 Signal resolution for a chirp signal with different window width parameter $\boldsymbol{a}$. (a-c) The signal time resolution for $f=0.25$. (d-f) The signal frequency resolution for $t=400$.

The normalized local signal segment is

$\eta_{t}(\tau)$

$$
\begin{aligned}
& =\frac{s(\tau) h(\tau-t) \exp \left\{j \omega t-j \sum_{m=2}^{M} \frac{\omega_{m-1}}{m !}(\tau-t)^{m}\right\}}{\sqrt{\int|s(\tau) h(\tau-t)|^{2} d \tau}} \\
& =\left(\frac{\alpha+a}{\pi}\right)^{1 / 4} \exp \left\{-\frac{a^{2} t^{2}}{2(\alpha+a)}\right\} \\
& \exp \left\{-\frac{(\alpha+a) \tau^{2}}{2}+a \tau t+j \sum_{m=1}^{M} \frac{a_{m-1} t^{m}}{m !}\right\} \\
& \exp \left\{j \omega t-j \sum_{m=2}^{M} \frac{\omega_{m-1}(\tau-t)^{m}}{m !}\right\},
\end{aligned}
$$

and

$\left|\eta_{t}(\tau)\right|^{2}=\left(\frac{\alpha+a}{\pi}\right)^{1 / 2} \exp \left\{-(\alpha+a)\left(\tau-\frac{a t}{\alpha+a}\right)^{2}\right\}$.

The mean time $\langle\tau\rangle_{t}$ and duration $T_{t}^{2}$ of the $M$ th-order LPFT for the $M$ th-order polynomial phase signal are the same as given in Section 4, that is,

$$
\langle\tau\rangle_{t}=\frac{a t}{a+\alpha}
$$


Table 2 Expressions of the uncertainty principles for the Mth-order LPFT

\begin{tabular}{lc}
\hline Type of uncertainty principle & Uncertainty product \\
\hline 1. Global uncertainty principle & $T_{\mathrm{LPP}}^{2} B_{\mathrm{LPP}}^{2} \geq 1$ \\
$\begin{array}{l}\text { 2. Local duration-conditional standard } \\
\text { deviation }\end{array}$ & $\sigma_{\omega \mid t}^{2} T_{t}^{2} \geq \frac{1}{4}$ \\
$\begin{array}{l}\text { 3. Local bandwidth-conditional standard } \\
\text { deviation }\end{array}$ & $\sigma_{t \mid \omega}^{2} B_{\omega}^{2} \geq \frac{1}{4}$ \\
$\begin{array}{l}\text { 4. Conditional standard deviations in time } \\
\text { and frequency }\end{array}$ & $\sigma_{t \mid \omega}^{2} \sigma_{\omega \mid t}^{2}>\frac{1}{4}$
\end{tabular}

and

$$
T_{t}^{2}=\frac{1}{2(a+\alpha)}
$$

When the parameters of the LPFT such as $\omega_{1}$, $\omega_{2}, \ldots, \omega_{M-1}$ are estimated correctly, that is, $\omega_{M-1}=$ $\Phi^{(M)}=a_{M-1}, \ldots, \omega_{m}=\Phi^{(m+1)}, \ldots, \omega_{1}=\Phi^{(2)}=$ $\sum_{m=1}^{M} \frac{a_{m-1} t^{m-2}}{(m-2) !}$, where the values of superscripts in $\Phi^{(m)}$ are the derivative orders of $\Phi$, we have

$$
\begin{aligned}
& \eta^{*}(\tau) \frac{d}{d \tau} \eta_{t}(\tau) \\
& =\left(\frac{\alpha+a}{\pi}\right) \exp \left\{-\frac{a^{2} t^{2}}{a+\alpha}\right\} \exp \left\{-(a+\alpha) \tau^{2}+2 a t \tau\right\} \\
& \cdot\left\{-(a+\alpha) \tau+a t+j \sum_{m=1}^{M} \frac{a_{m-1} t^{m-1}}{(m-1) !}\right\} .
\end{aligned}
$$

Therefore,

$$
\begin{aligned}
\langle\omega\rangle_{t} & =\left(\frac{\alpha+a}{\pi}\right) \exp \left\{-\frac{a^{2} t^{2}}{a+\alpha}\right\} \\
& \cdot \int \exp \left\{-(a+\alpha) \tau^{2}+2 a t \tau\right\} \\
& \cdot\left\{j(a+\alpha) \tau-j a t+\sum_{m=1}^{M} \frac{a_{m-1} t^{m-1}}{(m-1) !}\right\} d \tau \\
& =\sum_{m=1}^{M} \frac{a_{m-1} t^{m-1}}{(m-1) !} \\
& =\Phi^{(1)},
\end{aligned}
$$

which is the instantaneous frequency of the Mth-order polynomial phase signals.

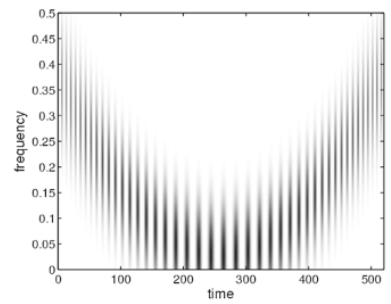

(a) LPP with $a=25$

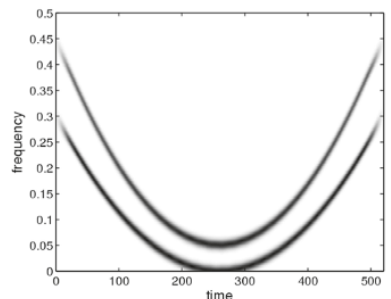

(b) LPP with $a=2.5$

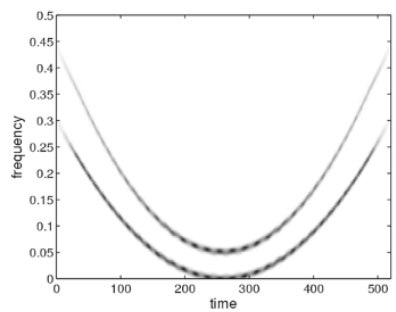

(c) LPP with $a=0.25$

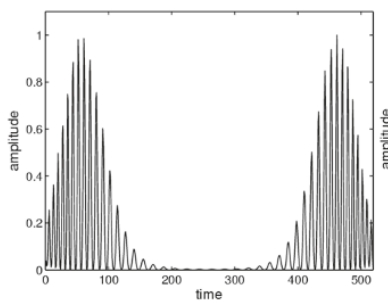

(d) time resolution, $a=25$

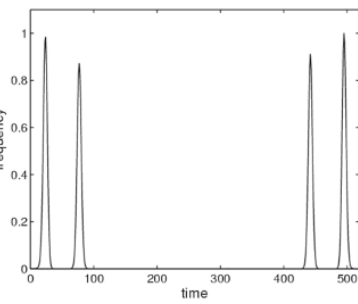

(e) time resolution, $a=2.5$

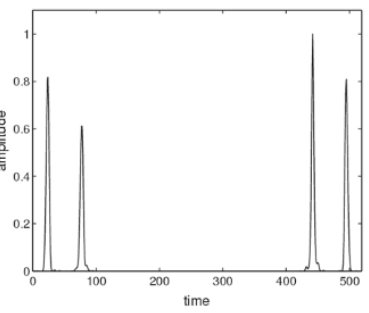

(f) time resolution, $a=0.25$

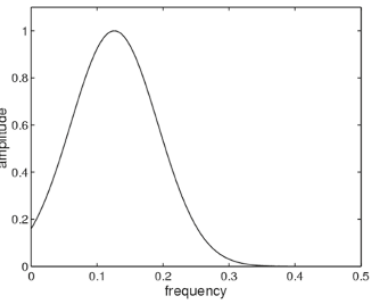

(g) frequency resolution, $a=25$

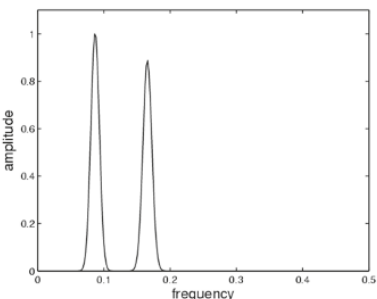

(h) frequency resolution, $a=2.5$

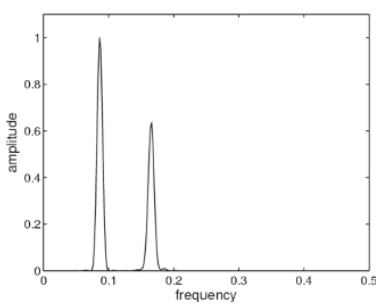

(i) frequency resolution, $a=0.25$

Figure 3 Signal resolution for a third order polynomial phase signal with different window width parameter $a$. (a)-(c): the third order LPP. (d)-(f): the signal time resolution for $f=0.25$. (g)-(i): the signal frequency resolution for $t=400$. 
Similar with $\omega_{M-1}=\Phi^{(M)}=a_{M-1}, \ldots, \omega_{m}=\Phi^{(m+1)}$, $\ldots, \omega_{1}=\Phi^{(2)}=\sum_{m=1}^{M} \frac{a_{m-1} t^{m-2}}{(m-2) !}$, we have

$$
\begin{aligned}
& \left\langle\omega^{2}\right\rangle=-\int \eta^{*}(\tau) \frac{d^{2} \eta_{t}(\tau)}{d \tau^{2}} d \tau \\
& =\int\left\{a+\alpha+\left[(a+\alpha) \tau-a t-j \sum_{m=1}^{M} \frac{a_{m-1} t^{m-1}}{(m-1) !}\right]^{2}\right\} \\
& \quad \cdot \exp \left\{-(a+\alpha) \tau^{2}+2 a t \tau\right\} d \tau \\
& =\frac{a+\alpha}{2}+\left[\sum_{m=1}^{M} \frac{a_{m-1} t^{m-1}}{(m-1) !}\right]^{2} .
\end{aligned}
$$

Based on the above derivation, we can conclude that if the coefficients of the polynomial phase are accurately estimated, the bandwidth obtained by the LPFTs of any order $M>1$ is

$$
\begin{aligned}
\sigma_{\omega \mid t}^{2}=B_{t}^{2} & =\left\langle\omega^{2}\right\rangle-\langle\omega\rangle_{t}^{2} \\
& =\frac{a+\alpha}{2} .
\end{aligned}
$$

Similarly, we can get that

$$
\begin{aligned}
B_{\omega}^{2} & =\frac{1}{2\left(\frac{1}{a}+\frac{1}{\alpha}\right)}, \\
\sigma_{t \mid \omega}^{2} & =\frac{1}{2}\left(\frac{1}{a}+\frac{1}{\alpha}\right) .
\end{aligned}
$$

Therefore, we have the global uncertainty principle as

$$
T_{\mathrm{LPP}}^{2} B_{\mathrm{LPP}}^{2}=\frac{1}{4}\left(\frac{1}{a}+\frac{1}{\alpha}\right)(a+\alpha) \geq 1,
$$

and

$$
\begin{aligned}
\sigma_{\omega \mid t}^{2} T_{t}^{2} & =\frac{1}{4} \\
\sigma_{t \mid \omega}^{2} B_{\omega}^{2} & =\frac{1}{4} \\
\sigma_{\omega \mid t}^{2} \sigma_{t \mid \omega}^{2} & =\frac{1}{4}(a+\alpha)\left(\frac{1}{a}+\frac{1}{\alpha}\right)>\frac{1}{4} \quad \text { as } \quad \alpha \rightarrow 0 .
\end{aligned}
$$

It means that for the higher-order polynomial phase signals segmented by a Gaussian window function, the uncertainty products of the Mth-order LPFT are also consistent with the theoretical analysis in Section 3 if the phase parameters are accurately estimated.

An example using the third-order LPFT to process the third-order polynomial phase signal is given in Figure 3a,b,c. Figure 3d,e,f shows the signal time resolution by sampling the LPP at a particular frequency instant $f=0.25$, and Figure 3g,h,i shows the signal frequency resolution by sampling the LPP at a particular time instant $t=400$. From Figure 3, we have similar observations to those from Figures 1 and 2 for the second-order
LPFT processing the chirp signal, that is, as the parameter $a$ decreases, the time resolution is decreased while the frequency resolution is increased.

\section{Conclusions}

In this paper, systematic analysis of the uncertainty principles of the LPFT are demonstrated to show that as a windowed transform, the LPFT is limited by the uncertainty principles, including the global uncertainty principle, the uncertainty principle of local duration and conditional standard deviation, the uncertainty principle of local bandwidth and conditional standard deviation, and the uncertainty principle of the conditional standard deviations in time and frequency. Explicit expressions of the uncertainty principles for the second-order LPFT are derived, and an example is given in which the uncertainty relations for a chirp signal is obtained to show that the relations match with the derived expressions. The uncertainty principles of $M$ th-order LPFT are also discussed to show that they are consistent with the theoretical analysis.

\section{Abbreviations}

LPFT: local polynomial Fourier transform; LPP: local polynomial periodogram; STFT: short-time Fourier transform.

\section{Competing interests}

The authors declare that they have no competing interests.

\section{Acknowledgement}

This research work is supported by the National Natural Science Foundation of China (No. 61102164), the Open Project of Zhejiang Key Laboratory for Signal Processing (ZJKL_4_SP - OP2013 - 02), and the Scientific Research Foundation for the Returned Overseas Chinese Scholars, State Education Ministry.

\section{Author details}

${ }^{1}$ School of Information Science and Engineering, 58 Haishu Road, Hangzhou Normal University, 311121 Hangzhou, China. ${ }^{2}$ School of Electronic and Electrical Engineering, 50 Nanyang Ave., Nangyang Technological University, Singapore 639798, Singapore.

Received: 8 September 2014 Accepted: 9 December 2014 Published: 16 December 2014

\section{References}

1. V Katkovnik, A new form of Fourier transform for time-frequency estimation. Signal Process. 47(2), 187-200 (1995)

2. D Gabor, textbf93, (26), Theory of communication. J. Inst. Electrical Eng, 429-457 (1946)

3. P Korn, Some uncertainty principles for time-frequency transforms of the Cohen class. IEEE Trans. Signal Process. 53(2), 523-527 (2005)

4. L Cohen, Time-Frequency Analysis. (Prentice Hall, NJ, 1995)

5. P Loughlin, L Cohen, The uncertainty principle: global, local, or both?. IEEE Trans. Signal Process. 52(5), 1218-1227 (2004)

6. V Katkovnik, Discrete-time local polynomial approximation of the instantaneous frequency. IEEE Trans. Signal Process. 46(10), 2626-2637 (1998)

7. X Li, G Bi, On uncertainty principle of the local polynomial Fourier transform. EURASIP J. Adv. Signal Process. 120 (2012)

8. L Cohen, The uncertainty principles of the short-time Fourier transform. Proc. SPIE. 2563, 80-90 (1995)

9. XXia, Discrete chirp-Fourier transform and its applications to chirp rate estimation. IEEE Trans. Signal Process. 48(11), 3122-3133 (2000)

10. X $\mathrm{LV}, \mathrm{G} B \mathrm{Bi}, \mathrm{C}$ Wan, $M$ Xing, Lv's distribution: principle, implementation, properties and performance. IEEE Trans. Signal Process. 59(8), 3576-3591 (2011) 
11. $X \mathrm{Li}, \mathrm{G} \mathrm{Bi}, \mathrm{Y} J \mathrm{u}$, Quantitative SNR analysis for ISAR imaging using local polynomial Fourier transform. IEEE Trans. Aerospace Electronic Syst. 45(3), 1241-1248 (2009)

12. I Djurovic, T Thayaparan, L Stankovic, Adaptive local polynomial Fourier transform in ISAR. EURASIP J. Appl. Signal Process. 2006, 1-15 (2006)

13. S Djukanovic, M Dakovic, L Stankovic, Local polynomial Fourier transform receiver for nonstationary interference excision in DSSS communications. IEEE Trans. Signal Process. 54(4), 1627-1636 (2008)

14. L Stankovic, S Djukanovic, Order adaptive local polynomial FT based interference rejection in spread spectrum communication systems. IEEE Trans. Instrum. Meas. 54(6), 2156-2162 (2005)

15. G Bi, X Li, C See, LFM signal detection using LPP-Hough transform. Signal Process. 91(6), 1432-1443 (2011)

16. V Katkovnic, A Gershman, A local polynomial approximation based beamforming for source localization and tracking in nonstationary environments. IEEE Signal Process. Lett. 7(1), 3-5 (2000)

17. X Li, G Bi, S Stankovic, A Zoubir, Local polynomial Fourier transform: a review on recent developments and applications. Signal Process. 91(6), 1370-1393 (2011)

18. YWei, GBi, Efficient analysis of time-varying muliticomponent signals with modified LPTFT. EURASIP J. Appl. Signal Process. 2005(1), 1261-1268 (2005)

doi:10.1186/1687-6180-2014-181

Cite this article as: $\mathrm{Li}$ and Bi: Systematic analysis of uncertainty principles of the local polynomial Fourier transform. EURASIP Journal on Advances in Signal Processing 2014 2014:181.

\section{Submit your manuscript to a SpringerOpen ${ }^{\circ}$ journal and benefit from:}

- Convenient online submission

- Rigorous peer review

- Immediate publication on acceptance

- Open access: articles freely available online

- High visibility within the field

- Retaining the copyright to your article

Submit your next manuscript at $\gg$ springeropen.com 